\title{
Femoral nerve paralysis due to pseudoaneurysm of the femoral artery after coronary angiography
}

\author{
Keywords: femoral nerve paralysis, coronary angiography, \\ pseudoaneurysm
}

\section{Introduction}

Neuropathic complications of cardiac catheterization are very low but through the increasing number of these interventions surgeons are going to be confronted with an increasing number of neuropathic complications which can be the only sign of an arteriovenous fistula or pseudoaneurysm. Femoral neuropathy is a rare complication of cardiac catheterization. ${ }^{1,2}$ Here we presented a 36-year-old male patient with femoral nerve paralysis due to pseudoaneurysm of the right femoral artery after coronary angiography.

Two months ago he underwent coronary angiography due to chest pain and syncope in a cardiology clinic two times with oneday interval. The patient applied to the emergency clinic with complaints of redness, severe pain and burning in the right groin and thigh 10 days after this intervention. The patient was operated on due to a pseudoaneurysm of the right femoral artery $(5.5 \times 2.5 \mathrm{~cm})$ and hematoma. A week after this operation the patient who was admitted to our outpatient clinic with the complaints of weakness in the right lower extremity, difficulty in walking, pain and burning in the right groin and thigh was hospitalized.

Through the musculoskeletal system examination the patient was walking independently without support, right antalgic and by stepping on the lateral side of the right foot. Right hip flexion strength was 2/5(According to Medical Research Council) and knee extension was 2/5. But Hip extension, abduction, and adduction could not be evaluated due to extreme pain. There was no patella reflex on the right. L1 and L2 dermatomes were found as hypoesthesic and L3 dermatome was found as anesthesic on the right. Allodynia and hyperalgesia were present. He had neuropathic pain according to Dolor Neuropatico 4(DN4 neuropathic pain questionnaire). Pain according to visual analog scale (VAS) was 7. He was a smoker (60packs/year) and $\mathrm{He}$ was under the medication including Atorvastatin Calcium $40 \mathrm{mg}$ 1x1, Metoprolol Succinate 50mg, Zofenopril Calcium 30mg, Acetylsalicylic Acid 100mg, ticagrelor 90mg, B1 vitamin, B6 vitamin, B12 vitamin and Purified Micronized Flavonoid Fraction 1000mg.

Sedimentation and $\mathrm{C}$ reactive protein were normal, leucocytosis and neutrophil dominance were present. The electroneurophysiological finding was acute sensorimotor axonal degeneration in the segment of the right femoral nerve below the inguinal ligament and acute demyelinating degeneration of the lateral femoral cutaneous nerve in the right side. On magnetic resonance imaging (MRI), after contrast injection peripheral enhanced fluid collection, inflammation, and bilateral inguinal lymphadenopathy were detected in the femoral artery-ven junction of the right inguinal region. Lumbar and sacral MRI was normal. However, pelvic MRI showed a smooth contouring cystic lesion $(26 \times 16 \mathrm{~mm})$ on the right inguinal region and several lymph nodes. Ultrasonography revealed anechoic cystic lesion (seroma or hematoma) and several lymph nodes in the right inguinal
Volume 3 Issue I - 2018

\author{
Canan Celik,' Ilker Ilhanli,' Fazil Kulakli,' \\ Adem Türköz,' Elif Uysal,' Özkan Özen, \\ Burcu Ünlü ${ }^{3}$ \\ 'Department of Physical Medicine and Rehabilitation, Giresun \\ University, Turkey \\ 2Department of Radiology, Alanya Alaaddin Keykubat University, \\ Antalya, Turkey \\ ${ }^{3}$ Department of Physical Medicine and Rehabilitation, Giresun \\ Physical Therapy and Rehabilitation Hospital,Turkey
}

\author{
Correspondence: Fazil Kulakli, Department of Physical \\ Medicine and Rehabilitation, Giresun University Prof. Dr. M. \\ İlhan Özdemir Training and Research Hospital, Giresun, Turkey, \\ Tel +904543101600, Fax +904543101699, \\ Email drfzl46@gmail.com
}

Received: December 09, 2017 | Published: January II, 2018

region, too. By infectious diseases clinic, ampicillin-sulbactam 6 gram/day i.v. was started empirically. Pregabalin $(300 \mathrm{mg} /$ day) and duloxetine $(30 \mathrm{mg} /$ day) were started for neuropathic pain. A rehabilitation program was started including electrical stimulation to hip flexor and knee extensor muscles, strengthening exercises and gait training and transcutaneous electrical nerve stimulation to the right hip and thigh. After 15 days, superficial tissue ultrasonography was repeated and reduction in the size of the anechoic cystic lesion in the right inguinal region was detected $(7.5 \times 2.4 \mathrm{~mm})$. There was no cystic lesion in the ultrasonographic control after one month.

Although, we considered the cause of neuropathy as pseudoaneurysm in our patient, traumatic neuropathy secondary to mechanical or digital pressure for achieving hemostasis can also occur after cardiac catheterization and should be kept in mind. ${ }^{3,4}$ Also, retroperitoneal hematoma following systemic anticoagulation therapy is one of the reasons for femoral neuropathy. ${ }^{5}$ Imaging procedures including MRI and ultrasonography are able to diagnose space consuming lesions. We conducted lumbar, sacral and pelvic MRI and ultrasonography of the region to exclude other pathologies. An electroneurophysiological examination is still the best way to diagnose the effected affected femoral nerve segment ${ }^{6}$ and we found acute sensorimotor axonal degeneration in the segment of the right femoral nerve below the inguinal ligament. Pregabalin ${ }^{7}$ and duloxetine ${ }^{8}$ are seen effective in neuropathic pain, and in our patient symptoms reduced by the combination of pregabalin and duloxetine. As it is mentioned by Kent et al. ${ }^{9}$ neuropathy after cardiac catheterization can be initially disabling, but it is usually completely reversible. By the 30 days rehabilitation program, our patient was recovered totally.

\section{Conclusion}

Pseudoaneurysm of the femoral artery after coronary angiography should be kept in mind in patients with weakness and neuropathic 
pain on the lower extremity due to femoral nerve paralysis. With the treatment of neuropathic pain and an effective rehabilitation program, a complete healing can be achieved as in our patient.

\section{Acknowledgements}

None.

\section{Conflict of interest}

The author declares no conflict of interest.

\section{References}

1. Jacobs MJ, Gregoric ID, Reul GJ. Profunda femoral artery pseudoaneurysm after percutaneous transluminal procedures manifested by neuropathy. J Cardiovasc Surg. 1992;33(6):729-731.

2. Kuruvilla A, Kuruttukulam G, Francis B. Femoral neuropathy following cardiac catheterization for balloon mitral valvotomy. International Journal of Cardiology. 1999;71(2):197-198.

3. Simon AW. Use of a mechanical pressure device for hemostasis following cardiac catheterization. Am J Crit Care. 1994;3(1):62-64.
4. Pradhan S, Gupta A. Iatrogenic median and femoral neuropathy. $J$ Assoc Phys India. 1995;43(2):141.

5. Warfel BS, Marini SG, Lachmann EA, et al. Delayed femoral nerve palsy following femoral vessel catheterization. Arch Phys Med Rehabil. 1993;74(11):1211-1215.

6. Abel NA, Januszewski J, Vivas AC, et al. Femoral nerve and lumbar plexus injury after minimally invasive lateral retroperitoneal transpsoas approach: electrodiagnostic prognostic indicators and a roadmap to recovery. Neurosurg Rev. 2017.

7. Sakai T, Murata H, Hara T. A case of scrotal pain associated with genitofemoral nerve injury following cystectomy. Journal of Clinical Anesthesia. 2016;32:150-152.

8. Kachko L, Ben ami S, Liberman A, et al. Duloxetine contributing to a successful multimodal treatment program for peripheral femoral neuropathy and comorbid 'reactive depression' in an adolescent. Pain res Manage. 2011;16(6):457-459.

9. Kent KC, Moscucci M, Gallagher SG, et al. Neuropathy after cardiac catheterization: incidence, clinical patterns and long term outcome. $J$ Vasc Surg. 1994;19(6):1008-1013. 